\title{
Lifelong Physical Activity and Cardiovascular Autonomic function in Mid-life
}

Antti M. Kiviniemi ${ }^{1 *}$, Nelli Perkiömäki ${ }^{1}$, Juha Auvinen ${ }^{2,3}$, Sauli Herrala ${ }^{2,3}$, Arto J. Hautala ${ }^{4}$, Riikka Ahola ${ }^{1,5}$, Tuija Tammelin ${ }^{6}$, Mikko P. Tulppo ${ }^{1}$, Marjo-Riitta Järvelinn ${ }^{2,3,7,8}$, Raija Korpelainen $^{1,2,9}$, Heikki V. Huikuri ${ }^{1}$

${ }^{1}$ Medical Research Center Oulu, Oulu University Hospital and University of Oulu; ${ }^{2}$ Center for Life Course Health Research, University of Oulu; ${ }^{3}$ Unit of Primary Care, Oulu University Hospital; ${ }^{4}$ Biomedical Engineering Research Group, University of Oulu; ${ }^{5}$ Research Unit of Medical Imaging, Physics and Technology, University of Oulu, Oulu, Finland; ${ }^{6}$ LIKES-Research Center for Sport and Health Sciences, Jyväskylä, Finland; ${ }^{7}$ Department of Epidemiology and Biostatistics, MRC-PHE Centre for Environment \& Health, School of Public Health, Imperial College London, London, UK; ${ }^{8}$ Biocenter Oulu, University of Oulu; and ${ }^{9}$ Department of Sports and Exercise Medicine, Oulu Deaconess Institute, Oulu, Finland Running head: Kiviniemi et al. Physical activity and autonomic function

*Correspondence: Antti M. Kiviniemi, Research Unit of Internal Medicine, Medical Research Center Oulu, PO Box 5000 (Kajaanintie 50), FI-90014 University of Oulu, Finland, email: antti.m.kiviniemi@oulu.fi, tel: +358 8315 4464, fax: +358 83155599

DISCLOSURE OF FUNDING: This work was supported by University of Oulu [grant number 24000692], Oulu University Hospital [grant number 24301140], European Regional Development Fund [grant number 539/2010 A31592], the Academy of Finland [grant numbers 267435, 285547], Ministry of Education and Culture [grant number 86/686/2014], the Sigrid Juselius Foundation, the Finnish Foundation for Cardiovascular Research, Helsinki, Finland, and the European Union's Horizon 2020 research and innovation programme [grant number 633595]. CONFLICT OF INTEREST: None. The results of the present study do not constitute endorsement by ACSM. 


\section{ABSTRACT}

Purpose: Physical activity (PA) associates with cardiovascular autonomic function but the relationship with lifelong PA is unclear. We hypothesized that lifelong PA would associate with cardiovascular autonomic function in mid-life. Methods: At the age of 46, the subjects of the prospective Northern Finland Birth Cohort 1966 were invited to examinations where vagally-mediated heart rate variability (rMSSD) and cross-spectral baroreflex sensitivity (BRS) were analysed from 3-min recordings of ECG and blood pressure in seated and standing positions. Three lifelong PA trajectory groups (active, semi-active and inactive) were formed according to their self-reported frequencies of participation in PA at the ages of 14, 31 and 46. Finally, 1,283 men and 1,779 women without cardiorespiratory diseases and diabetes had complete data on lifelong PA, covariates and rMSSD, and 662 men and 807 women for BRS. Results: In both sexes and measurement conditions, the active $(\mathrm{p}<0.01)$ and semi-active groups $(\mathrm{p}<0.05)$ had greater rMSSD than the inactive group, and the highest BRS was observed in the active group (ANOVA p=0.001-0.032). In men, these differences were not significant when adjusted for 46-year lifestyle (smoking, alcohol consumption, sleep, and sitting time), body mass index, waist-hip-ratio, blood pressure, lipid and glucose status. In women, lifelong PA remained a significant independent determinant of seated and standing rMSSD and standing BRS. Conclusion: Higher lifelong PA was associated with better cardiovascular autonomic function in mid-life. In women, this effect was independent but in men, it seemed to be mediated by the other lifestyle and cardiometabolic factors.

Key Words: exercise; autonomic nervous system; heart rate variability; baroreceptors 


\section{Introduction}

Paragraph 1 - Physical inactivity is an important risk factor for the development of various cardiometabolic diseases. Importantly, physical activity (PA) has been associated positively with cardiac vagal activity, as measured by heart rate variability (HRV) and baroreflex sensitivity $(\mathrm{BRS})(5,15,18,21,24)$. Cardiovascular autonomic function is known to be related to cardiometabolic risk factors $(11,30)$, and its improvement with increased PA has been postulated as one potential mechanism that leads to reduced cardiovascular risk, independently of traditional risk markers (9). Impaired cardiovascular autonomic function, manifested as depressed vagal and augmented sympathetic activity, has been shown to increase the susceptibility to cardiac events and death in various human populations $(13,14)$.

Paragraph 2 - The effects of lifelong PA on HRV in retrospective studies are inconclusive $(10,32)$ and, as far as we are aware, there are no studies which have investigated the relationship between lifelong PA and BRS. Notably, some cross-sectional studies have not detected any impact of PA on cardiovascular autonomic function that is independent of demographic, metabolic and genetic factors uniformly in both sexes $(3,6,11,25)$. The strong age- and sex-dependency of cardiovascular autonomic function may attenuate the contribution of PA to HRV and BRS $(6,11,30)$.

Paragraph 3 - The purpose of the present study was to determine whether lifelong PA contributes independently to cardiovascular autonomic function in middle-aged men and women in large and prospective Northern Finland Birth Cohort 1966 (NFBC1966). We hypothesized that high level of lifelong PA would be associated with better cardiovascular autonomic function in these now middle-aged subjects of NFBC1966.

\section{Methods}


Paragraph 4-Subjects: The subjects whose expected date of birth fell between January $1^{\text {st }}$ and December $31^{\text {st }} 1966$ in Northern Finland (96.3\% of all 1966 births, $n=12,058$ live births) were included in the prospective NFBC1966-study. Since their mothers' recruitment during their first visit to the maternity health centers, data have been collected on the subjects' health, lifestyle and socioeconomic status. The study was conducted according the Declaration of Helsinki and approved by the Ethical Committee of the Northern Ostrobothnia Hospital District in Oulu, Finland. The subjects and their parents provided their written informed consent for the study.

Paragraph 5 - Protocol: Postal questionnaires enquiring about the participant's health status and lifestyle was conducted in 1980, 1997-1998, and 2012-2014, i.e. when the participants were aged 14, 31 and 46 years. The response rate was $97 \%$ at age $14(n=11,399)$, $75 \%$ at age $31(n=8,767)$ and $66 \%$ at age $46(n=6,825)$. Subjects who were living at known addresses in Finland $(n=10,282)$, were invited to attend the clinical examinations in one of three laboratory units (Oulu, Southern and Northern Finland). A total of 5,861 (57\%) subjects participated in clinical examinations between April 2012 and March 2014. The subjects entered the laboratory between 7:00 and 11:00 a.m. after overnight fasting (12 hours) and abstained from smoking and drinking coffee during the examination day. Venous blood samples were drawn for the analysis of glycemic and lipid status. Seated systolic (SBP) and diastolic blood pressures (DBP) were measured three times (the two lowest values averaged, Omron M10, Omron Healthcare, Kyoto, Japan) after 15 minutes of rest. After anthropometric measurements and other examinations, the participants had a light meal 60-90 min before the assessments of cardiovascular autonomic function. On a separate day, an oral glucose tolerance test was conducted according to the recommendations in those participants without medication for diabetes (1). 
Paragraph 6 - Measurement of cardiovascular autonomic function: Each participant sat down on a chair to allow instrumentation and a review of the protocol. A heart rate (HR) monitor (RS800CX, Polar Electro Oy, Kempele, Finland) was used to record R-R intervals (RRi). In half of the participants (Oulu laboratory unit) spontaneous BRS was also assessed. Standard lead-II ECG (Cardiolife, Nihon Kohden, Tokyo, Japan), breathing frequency (MLT415/D, Nasal Temperature Probe, ADInstruments, Bella Vista, New South Wales, Australia), and blood pressure (BP) by finger photoplethysmography (Nexfin, BMEYE Medical Systems, Amsterdam, the Netherlands) were recorded with a sampling frequency of $1,000 \mathrm{~Hz}$ (PowerLab 8/35, ADInstruments). The finger cuff was adjusted so that SBP and DBP assessed by finger photoplethysmography (left arm, supported by an arm sling) did not differ by more than $10 \mathrm{mmHg}$ from the values measured by automated sphygmomanometer (right arm, Omron M10). Physiological calibration of finger BP was then turned off. After these procedures (5-10 $\mathrm{min})$, there was at least 1-min period allowing stabilization of HR before the recording that included $3 \mathrm{~min}$ at the seated and $3 \mathrm{~min}$ at standing position while breathing spontaneously. Standing position was included because the measures of BRS reflect baroreflex physiology better during upright position and are more reproducible $(8,28)$. The first $150 \mathrm{~s}$ of recording in the seated position and the last $150 \mathrm{~s}$ in the standing position were analyzed.

Paragraph 7 - Analysis of heart rate variability: Artefacts and ectopic beats were removed and replaced by the local average (Hearts 1.2, University of Oulu, Oulu, Finland). However, sequences with $\geq 10$ consecutive beats of noise or ectopic beats were totally deleted. The RRi series with $\geq 80 \%$ accepted data were included in the analyses. A total of 5,679 subjects took part in the RRi recordings and of these 5,473 (96\%) had eligible HRV data for both phases of the protocol (seated and standing). Mean HR and root mean square of successive differences in RRi (rMSSD, ms), a robust measure of cardiac vagal activity, were analyzed along with 
short-term fractal-like scaling exponent $\left(\alpha_{1}\right)$, which was used as an estimate of sympathovagal balance $(20,27,31)$.

Paragraph 8 - Analysis of baroreflex sensitivity: Continuous ECG, BP and respiration signals were imported to a custom-made standalone Matlab-based software (Biosignal Processing Team, University of Oulu, Oulu, Finland) where RRi and SBP values were extracted (12). Artefacts and ectopic beats were replaced using linear interpolation $(<5 \%$ for accepted recording) and thereafter, resampled at $2 \mathrm{~Hz}$ and detrended $(<0.04 \mathrm{~Hz}$, SavitzkyGolay method). A fast Fourier transform (Welch method, segments of 128 samples with 50\% overlap) was performed to analyze low frequency (LF, 0.04-0.15 Hz) power of RRi and SBP oscillations for subsequent analysis of BRS by the alpha method, if sufficient coherence $(\geq 0.5)$ between LF oscillations in RRi and SBP was verified. Out of 2,726 recordings, BRS was successfully calculated for 2,599 subjects (95\%) in both the seated and standing positions.

Paragraph 9 - Assessment of physical activity: PA was self-reported at 14, 31 and 46 years of age ( $\mathrm{PA}_{14 y}, \mathrm{PA}_{31 y}$ and $\left.\mathrm{PA}_{46 \mathrm{y}}\right)$. At the age of 14 , the subjects were asked how often they participated in sports after school hours with the following alternatives 1) daily, 2) every other day, 3) twice a week, 4) once a week, 5) every other week, 6) once a month and 7) generally not at all. The answer options 6 and 7 were combined. At the ages of 31 and 46, the subjects were asked how often they participated in brisk physical activity/exercise during their leisuretime. The term 'brisk' was defined as physical activity causing at least some sweating and getting out of breath, corresponding to moderate-to-vigorous intensity. The six response alternatives were 1) daily, 2) 4-6 times a week, 3) 2-3 times a week, 4) once a week, 5) 2-3 times a month, and 6) once a month or less often. For the age-specific PA variables, the two consecutive answers were combined.

Paragraph 10 - Latent Class Analysis (LCA) was used to obtain clusters in which the individuals had a similar profile of PA from adolescence to middle-age (Auvinen et al. 
manuscript). Three lifelong PA trajectory groups (active, semi-active and inactive) were formed according to the self-reported frequency of PA at 14, 31 and 46 years of age (PA Life). . In LCA, the number of clusters is increased until the most appropriate model is found (19). We assessed models with cluster number of one to seven and determined the best-fitting cluster solution of these candidates by calculating the Bayesian Information Criterion. We considered the interpretability of the classification, the conceptual meaningfulness of the models, and the sizes of the subgroups while choosing the best solution (17). The clustering was done for all the applicable subjects and both sexes together, not only for those subjects with measured HRV and BRS.

Paragraph 11 - Lifestyle factors at age 46: Based on questionnaire, subjects were defined as current smokers if they smoked regularly $\geq 1$ cigarrette/day on $\geq 2$ days/week. The amount of alcohol consumed per day was estimated from the questions measuring the frequency and the usual amount beverages consumed on one occasion. The subjects were then categorized into two groups based on the highest sex-specific deciles rounded to the closest 10 $\mathrm{g} / \mathrm{d}$ (40 g/d for men, $20 \mathrm{~g} / \mathrm{d}$ for women). Total sitting time during waking hours was established by asking the subjects how many hours on average they sat on weekdays (at work, at home, in vehicle and elsewhere) and the sum of sitting times was dichotomized based on previously reported cutoff value of $11 \mathrm{~h} / \mathrm{d}$ (33). To evaluate the sufficiency of sleep, the subjects were asked how tired they felt in the morning during the first half hour after awakening (very/somewhat tired=insufficient, somewhat/well rested=sufficient).

Paragraph 12 - Inclusions/Exclusions: Among subjects with eligible HRV data $(\mathrm{n}=5,473), \mathrm{PA}_{\text {Life }}$ could be determined in 4,330 subjects. Among them, 255 patients with previous or newly diagnosed diabetes $(1,34)$, cardiac $(n=88)$ or respiratory disease $(n=246$, including asthma), antihypertensive medication $(\mathrm{n}=570)$ or missing data for any covariate 
$(n=125)$ were excluded. The final population included 1283 men and 1779 women for HRV, 662 men and 807 women for BRS (Table 1, Supplementary table 1).

Paragraph 13 - Statistical analysis: Gaussian distributions of the dependent variables were verified by visual inspection of the histograms. In case of skewed distribution, the variable was transformed into a natural logarithm before statistical analyses (rMSSD and BRS). Oneway ANOVA was used to compare the PA groups and sexes which was followed by Bonferroni's post hoc test when applicable. Sex-differences in categorical variables were assessed by Chi-square. The linearity and collinearity of associations were assessed by the linear and quadratic regression models or contrasts estimated by ANOVA. Thereafter, the multivariate linear regression analysis (enter method) was employed to determine the total contribution of the most potential confounders (Block I: smoking, alcohol consumption, sitting time, sufficiency of sleep, body mass index) and mediators (Block II: waist-hip-ratio, brachial systolic and diastolic blood pressure, glycated hemoglobin, fasting plasma glucose, serum total and high-density lipoprotein cholesterol and triglycerides) of the relationship between PA and cardiac autonomic function. Low-density lipoprotein cholesterol was excluded from Block II due to its significant collinearity with total cholesterol. Subsequently, independent association of PA to autonomic measures were established (Block I+PA, Block I+II+PA). Complementary step- and blockwise multivariate regression (Block I [incl. PA Life $\left._{3}+\mathrm{II}\right)$ was applied to establish the most significant associations of lifestyle and cardiometabolic factors to autonomic measures (data supplements). The data were analyzed using SPSS software (IBM SPSS Statistics 21, IBM Corp., New York). A p-value <0.05 was considered significant.

\section{Results}

Paragraph 14 - In men, univariate analysis detected that $\mathrm{PA}_{\text {Life }}$ was associated with all measures of cardiovascular autonomic function, except for $\alpha_{1}$ in the seated position (Table 2, 
Figure 1). A similar pattern was observed with $\mathrm{PA}_{46} \mathrm{y}$, whereas the impact of $\mathrm{PA}_{31 \mathrm{y}}$ was only observed in seated HR, seated rMMSD, and standing HR. In the multivariate analysis (Block I+PA $\left.A_{\text {Life}}\right), \mathrm{PA}_{\text {Life }}$ was significantly associated with $\mathrm{HR}$ in the seated and standing position but not with the other measures of autonomic function (Table 2). E.g. current smoking was more strongly associated with outcomes in autonomic function than $\mathrm{PA}_{\text {Life }}$ in men (Supplementary table 2). However, PA46y was independently related to the measures of cardiovascular autonomic function (Table 2).

Paragraph 15 - In women, $\mathrm{PA}_{\text {Life }}$ was related to all measures of cardiovascular autonomic function in both the seated and standing positions, with the exception of $\alpha_{1}$ (Table 3, Figure 2). PA46y was associated with seated BRS approaching borderline statistical significance $(\mathrm{p}=0.075)$. In the multivariate analysis $(\mathrm{Block} \mathrm{I}+\mathrm{PA}$ Life), all significant univariate associations between $\mathrm{PA}_{\mathrm{Life}}$ and cardiovascular autonomic function remained significant, with exception of the seated BRS (Table 3). Body mass index and BP were also potent factors underlying the variance in autonomic function (Supplementary table 3).

\section{Discussion}

Paragraph 16 - This is the first prospective study to reveal that lifelong PA associates with cardiovascular autonomic function, as measured by HRV and BRS, in a middle-aged population. In women, lifelong PA was independently associated with vagally-mediated HRV and BRS. In men, however, this association was not independent of the other lifestyle and cardiometabolic factors that may mediate the autonomic adaptations to lifelong PA. These findings underscore the importance of long-term PA in prevention of autonomic dysfunction and related cardiovascular risk.

Paragraph 17 - The present study revealed independent association between current level of PA at middle-age, and cardiovascular autonomic function in both men and women. In 
contrast to some previous findings $(3,6,11,25)$, the present study supports the proposal that PA exerts a significant impact of cardiovascular autonomic function that is not completely explained by the other cardiometabolic and lifestyle factors $(5,9,15,18,21,24)$. The intensity of the PA may explain some of the previous conflicting findings. A less significant impact of PA on cardiovascular autonomic function has been observed in those studies that did not estimate the amount of PA at higher intensities $(3,11,25)$, whereas investigations detecting positive associations between cardiovascular autonomic function and PA have found that the relationship exists particularly with moderate-to-vigorous PA $(5,21)$.

Paragraph 18 - The present association between lifelong PA and cardiovascular autonomic function indicated that there are important sex-related factors involved. Uusitalo et al. have reported that lifelong PA was not independently related to cardiovascular autonomic function in middle-aged men (32). The current documentation is in good agreement with the findings by Kaikkonen et al. where lifelong PA was independently associated with HRV in obese, mostly female individuals (10). One possible reason for these sex-specific differences may be autonomic function exhibits greater adaptability to prevailing level of PA in middleaged men than women (2). This theory is supported by our data showing that also the other lifestyle factors at middle-age were more closely related to cardiovascular autonomic function in men than in women, particularly smoking and sufficiency of sleep (Supplementary tables 2 and 3). We also observed that PA at age of 31 was a more potent determinant of cardiovascular autonomic function in women than in men, which also supports this hypothesis. While the earlier PA affects the PA at later life (26), it is important to note PA $14 \mathrm{y}$ was not associated with cardiovascular autonomic function at mid-life. Therefore, PA during adulthood seems to be the key for the present observations. It is noteworthy that we cannot establish the causality between PA and cardiac autonomic function. Therefore, there may be factors during the growth and 
aging affecting both long-term PA and the outcomes in autonomic nervous system that remain to be established.

Paragraph 19 - Age and sex are the two most important modifiers of cardiovascular autonomic function $(6,11,30)$. Along with large sample size, the strength of the present study is that age was strictly controlled in the sex-specific analyses. This, however, also explains that in total, independent factors accounted for only $~ 20 \%$ of variance in HRV and BRS in men, and even less in women. However, this is at least as large as age- and sex-adjusted contribution of the other demographic and lifestyle factors on HRV and BRS in previous studies $(6,11,25$, $32)$.

Paragraph 20 - The present study provides novel information about the effects of prospectively assessed lifelong PA on BRS, which is known to be a strong determinant of cardiovascular risk $(13,14)$. PA $A_{\text {Life }}$ was associated with BRS in both sexes, but associated independently only in women when BRS was assessed in the standing position. Notably, spontaneous BRS method reflects baroreflex physiology with better reproducibility, particularly when evaluated in the standing position $(8,28)$. Like HRV, BRS has been typically measured at supine rest why the results may not be fully comparable with the previous reports. In the present study, seated BRS and rMMSD values were lower than those reported previously from the recordings at supine rest in comparable populations $(11,23)$. Importantly, BRS reflects autonomic outflow to sinoatrial node in relation to the sensation of arterial transmural pressure by baroreceptors that is related to mechanical properties of the vessel (16) that may improve with exercise training (22).

Paragraph 21 - Fractal-like scaling exponent $\alpha_{1}$, an estimate of sympathovagal balance, was almost totally unrelated to any measured variable. While Fagard et al. have reported modest effects of PA on the low-to-high frequency ratio (6), a controversial measure of sympathovagal balance (7), several studies failed to confirm this association $(10,25,30,32)$. 
However, Kaikkonen et al. observed that physical fitness was related to the low-to-high frequency in the obese individuals but, as with the present fractal $\alpha_{1}$, the contribution of the other determinants remained low (10). While the assessments of sympathetic activity and sympathovagal balance by HRV methods are susceptible (7), the impact of lifelong PA on these features of autonomic regulation remains to be determined.

Paragraph 22 - Body mass index and BP were important factors underlying variance in HRV and BRS in both sexes although the former's independent value decreased when including cardiometabolic markers in the model (Supplementary tables 2 and 3). This suggests that lifestyle changes targeting on these risk factors may also benefit in terms cardiac autonomic function. For example, a unit decrease in rMSSD (ln) has corresponded $\sim 70 \%$ increase in adjusted risk for cardiac event during the mean follow-up of 3.5 years in middleaged general population (29). When assuming causality, the present results suggest that the cardiac risk related decreased rMSSD might halve by improving all unhealthy habits in men, potential effects of lifestyle changes being lesser in women. However, it must be acknowledged that the effects of lifestyle factors on cardiac morbidities are not exclusively mediated via autonomic nervous system.

\section{Study limitations}

Paragraph 23 - The HRV analysis is from the short-term recordings are less reproducible than longer-term ambulatory recordings (4). For example, cardiovascular autonomic function is affected by the time from the previous meal, which although controlled, was relatively short. This was a tradeoff related to other competing priorities resulting in some compromises in the extensive protocol of NFBC1966-study. Spontaneous breathing may confound the spectral analysis of HRV and BRS (20). In subjects with a breathing signal $(n=1,441), \sim 10 \%$ had a breathing frequency at LF but this was not associated with PA. Moreover, rMSSD is less 
vulnerable to slow breathing than the spectral indices. Instead of objective measurements, the lifelong PA and all the other lifestyle factors, including sufficiency sleep and sitting time, were based on self-report. Nevertheless, the present PA assessment was simple and based on the one question at each age, although quite infrequently, being easily reproducible. Finally, the population may not fully represent the whole NFBC1966 due incomplete participation to follow-up measurements and exclusions by cardiorespiratory and metabolic diseases and medications affecting autonomic function.

\section{Conclusion}

Paragraph 24 - High level of lifelong physical activity was independently related to cardiovascular autonomic function in middle-aged women. In men, this association was not independent of other lifestyle and health related factors that, in turn, may mediate the effects of lifelong physical activity on cardiovascular autonomic function. Notably, current physical activity was independently related to cardiac autonomic function also in men. The present findings highlight the importance of lifelong physical activity in preventing cardiac events mediated by impaired cardiovascular autonomic function; particularly among women, in whom physical activity seems to be the most important target for lifestyle intervention in this respect.

\section{ACKNOWLEDGMENTS}

This work was supported by University of Oulu [grant number 24000692], Oulu University Hospital [grant number 24301140], European Regional Development Fund [grant number 539/2010 A31592], the Academy of Finland [grant numbers 267435, 285547], Ministry of Education and Culture [grant number 86/686/2014], the Sigrid Juselius Foundation, the Finnish Foundation for Cardiovascular Research, Helsinki, Finland, and the European Union's Horizon 2020 research and innovation programme [grant number 633595]. We thank the late professor 
Paula Rantakallio (launch of NFBC1966), the participants in the 46y study and the NFBC project center. Meri-Maija Ollila, BM, is gratefully acknowledged for her help with processing of HRV data.

\section{CONFLICT OF INTEREST}

None. The results of the present study do not constitute endorsement by ACSM.

\section{FIGURE LEGENDS}

Figure 1. The root mean square of the successive differences in R-R intervals (rMSSD, a, c) and baroreflex sensitivity (BRS, b, d) across the groups based on life course trajectories of physical activity (PA $\left.\mathrm{PAfe}_{\mathrm{Lif}}\right)$ before $(\mathrm{a}-\mathrm{b})$ and after adjustments for lifestyle and laboratory markers at age of 46 (c-d) in men. Error bars are standard errors of means.

Figure 2. The root mean square of the successive differences in R-R intervals (rMSSD, a, c) and baroreflex sensitivity (BRS, b, d) across the groups based on life course trajectories of physical activity $\left(\mathrm{PA}_{\mathrm{Life}}\right)$ before $(\mathrm{a}-\mathrm{b})$ and after adjustments for lifestyle and laboratory markers at age of $46(\mathrm{c}-\mathrm{d})$ in women. Error bars are standard errors of means.

\section{REFERENCES}

1. Alberti KG, Zimmet PZ. Definition, diagnosis and classification of diabetes mellitus and its complications. Part 1: diagnosis and classification of diabetes mellitus provisional report of a WHO consultation. Diabet Med. 1998;15(7):539-553.

2. Carter JB, Banister EW, Blaber AP. The effect of age and gender on heart rate variability after endurance training. Med Sci Sports Exerc. 2003;35(8):1333-1340. 
3. De Geus EJ, Boomsma DI, Snieder H. Genetic correlation of exercise with heart rate and respiratory sinus arrhythmia. Med Sci Sports Exerc. 2003;35(8):1287-1295.

4. Dekker JM, de Vries EL, Lengton RR, Schouten EG, Swenne CA, Maan A. Reproducibility and comparability of short- and long-term heart rate variability measures in healthy young men. Annals of Noninvasive Electrocardiology. 1996;1(3):287-292.

5. Dietrich DF, Schindler C, Schwartz J, et al. Heart rate variability in an ageing population and its association with lifestyle and cardiovascular risk factors: results of the SAPALDIA study. Europace. 2006;8(7):521-529.

6. Fagard RH, Pardaens K, Staessen JA. Influence of demographic, anthropometric and lifestyle characteristics on heart rate and its variability in the population. J Hypertens. 1999;17(11):1589-1599.

7. Heathers JA. Sympathovagal balance from heart rate variability: an obituary. Exp Physiol. 2012;97(4):556.

8. Herpin D, Ragot S. Mid- and long-term reproducibility of noninvasive measurements of spontaneous arterial baroreflex sensitivity in healthy volunteers. Am J Hypertens. 1997;10(7 Pt 1):790-797.

9. Joyner MJ, Green DJ. Exercise protects the cardiovascular system: effects beyond traditional risk factors. J Physiol. 2009;587(Pt 23):5551-5558.

10. Kaikkonen KM, Korpelainen RI, Tulppo MP, et al. Physical activity and aerobic fitness are positively associated with heart rate variability in obese adults. $J$ Phys Act Health. 2014;11(8):1614-1621.

11. Kardos A, Watterich G, de Menezes R, Csanady M, Casadei B, Rudas L. Determinants of spontaneous baroreflex sensitivity in a healthy working population. Hypertension. 2001;37(3):911-916. 
12. Kiviniemi AM, Tiinanen S, Hautala AJ, et al. Frequency of slow oscillations in arterial pressure and R-R intervals during muscle metaboreflex activation. Auton Neurosci. 2010;152(1-2):88-95.

13. Kiviniemi AM, Tulppo MP, Hautala AJ, et al. Prognostic significance of impaired baroreflex sensitivity assessed from Phase IV of the Valsalva maneuver in a populationbased sample of middle-aged subjects. Am J Cardiol. 2014;114(4):571-576.

14. La Rovere MT, Bigger JT, Jr., Marcus FI, Mortara A, Schwartz PJ. Baroreflex sensitivity and heart-rate variability in prediction of total cardiac mortality after myocardial infarction. ATRAMI (Autonomic Tone and Reflexes After Myocardial Infarction) Investigators. Lancet. 1998;351(9101):478-484.

15. La Rovere MT, Pinna GD. Beneficial effects of physical activity on baroreflex control in the elderly. Ann Noninvasive Electrocardiol. 2014;19(4):303-310.

16. Lucini D, Palombo C, Malacarne M, Pagani M. Relationship between carotid artery mechanics and the spontaneous baroreflex: a noninvasive investigation in normal humans. J Hypertens. 2012;30(9):1809-1816.

17. Marsh HW, Ludtke O, Trautwein U, Morin AJS. Classical Latent Profile Analysis of Academic Self-Concept Dimensions: Synergy of Person- and Variable-Centered Approaches to Theoretical Models of Self-Concept. Structural Equation Modeling-a Multidisciplinary Journal. 2009;16(2):191-225.

18. Molgaard H, Hermansen K, Bjerregaard P. Spectral components of short-term RR interval variability in healthy subjects and effects of risk factors. Eur Heart J. 1994;15(9):1174-1183.

19. Nylund KL, Asparouhov T, Muthen BO. Deciding on the number of classes in latent class analysis and growth mixture modeling: A Monte Carlo simulation study. Structural Equation Modeling-a Multidisciplinary Journal. 2007;14(4):535-569. 
20. Penttilä J, Helminen A, Jartti T, et al. Time domain, geometrical and frequency domain analysis of cardiac vagal outflow: effects of various respiratory patterns. Clin Physiol. 2001;21(3):365-376.

21. Rennie KL, Hemingway H, Kumari M, Brunner E, Malik M, Marmot M. Effects of moderate and vigorous physical activity on heart rate variability in a British study of civil servants. Am J Epidemiol. 2003;158(2):135-143.

22. Seals DR, Desouza CA, Donato AJ, Tanaka H. Habitual exercise and arterial aging. $J$ Appl Physiol (1985). 2008;105(4):1323-1332.

23. Sinnreich R, Friedlander Y, Luria MH, Sapoznikov D, Kark JD. Inheritance of heart rate variability: the kibbutzim family study. Hum Genet. 1999;105(6):654-661.

24. Soares-Miranda L, Sattelmair J, Chaves P, et al. Physical activity and heart rate variability in older adults: the Cardiovascular Health Study. Circulation. 2014;129(21):2100-2110.

25. Stolarz K, Staessen JA, Kuznetsova T, et al. Host and environmental determinants of heart rate and heart rate variability in four European populations. $J$ Hypertens. 2003;21(3):525-535.

26. Tammelin T, Nayha S, Hills AP, Jarvelin MR. Adolescent participation in sports and adult physical activity. Am J Prev Med. 2003;24(1):22-28.

27. Task Force of the European Society of Cardiology and the North American Society of Pacing and Electrophysiology. Heart rate variability: standards of measurement, physiological interpretation and clinical use. Circulation. 1996;93(5):1043-1065.

28. Taylor JA, Eckberg DL. Fundamental relations between short-term RR interval and arterial pressure oscillations in humans. Circulation. 1996;93(8):1527-1532. 
29. Tsuji H, Larson MG, Venditti FJ, Jr., et al. Impact of reduced heart rate variability on risk for cardiac events. The Framingham Heart Study. Circulation. 1996;94(11):28502855.

30. Tsuji H, Venditti FJ, Jr., Manders ES, et al. Determinants of heart rate variability. J Am Coll Cardiol. 1996;28(6):1539-1546.

31. Tulppo MP, Kiviniemi AM, Hautala AJ, et al. Physiological background of the loss of fractal heart rate dynamics. Circulation. 2005;112(3):314-319.

32. Uusitalo AL, Vanninen E, Levalahti E, Battie MC, Videman T, Kaprio J. Role of genetic and environmental influences on heart rate variability in middle-aged men. Am J Physiol Heart Circ Physiol. 2007;293(2):H1013-1022.

33. van der Ploeg HP, Chey T, Korda RJ, Banks E, Bauman A. Sitting time and all-cause mortality risk in 222497 Australian adults. Arch Intern Med. 2012;172(6):494-500.

34. World Health Organization (WHO). Use of glycated haemoglobin (HbA1c) in the diagnosis of mellitus. Avabetes at: http://www.who.int/diabetes/publications/report-hba1c_2011.pdf Accessed 1.11.2015, 2011. 
Table 1. Characteristics of the study population at age of 46 .

\begin{tabular}{|c|c|c|c|}
\hline & $\begin{array}{c}\text { Men } \\
n=1283\end{array}$ & $\begin{array}{l}\text { Women } \\
\mathrm{n}=1779\end{array}$ & $\begin{array}{c}\mathrm{p} \\
\text { Value }\end{array}$ \\
\hline \multicolumn{4}{|l|}{ Lifestyle } \\
\hline Current smoker, $\mathrm{n}$ & $217(17)$ & $239(13)$ & 0.008 \\
\hline Alcohol consumption $\geq 40 / 20 \mathrm{~g} \cdot \mathrm{d}^{-1}(\mathrm{M} / \mathrm{F}), \mathrm{n}$ & $102(8)$ & $129(7)$ & - \\
\hline Insufficient sleep, $\mathrm{n}$ & $374(29)$ & $540(30)$ & 0.473 \\
\hline Sitting time $\geq 11 \mathrm{~h} \cdot \mathrm{d}^{-1}, \mathrm{n}$ & $203(16)$ & $165(9)$ & $<0.001$ \\
\hline \multicolumn{4}{|l|}{ Laboratory measurements } \\
\hline Systolic blood pressure, $\mathrm{mmHg}$ & $127.5(13.5)$ & $117.0(14.9)$ & $<0.001$ \\
\hline Diastolic blood pressure, $\mathrm{mmHg}$ & $84.8(9.8)$ & $80.4(10.3)$ & $<0.001$ \\
\hline Body mass index, $\mathrm{kg} \cdot \mathrm{m}^{-2}$ & $26.6(3.8)$ & $25.6(4.7)$ & $<0.001$ \\
\hline Waist-hip-ratio & $0.97(0.06)$ & $0.86(0.06)$ & $<0.001$ \\
\hline $\mathrm{HbA} 1 \mathrm{c}, \%$ & $5.46(0.34)$ & $5.38(0.35)$ & $<0.001$ \\
\hline Plasma glucose, $\mathrm{mmol} \cdot \mathrm{L}^{-1}$ & $5.57(0.46)$ & $5.25(0.45)$ & $<0.001$ \\
\hline Total cholesterol, $\mathrm{mmol} \cdot \mathrm{L}^{-1}$ & $5.56(0.96)$ & $5.13(0.84)$ & $<0.001$ \\
\hline LDL cholesterol, $\mathrm{mmol} \cdot \mathrm{L}^{-1}$ & $3.74(0.91)$ & $3.17(0.84)$ & $<0.001$ \\
\hline HDL cholesterol, $\mathrm{mmol} \cdot \mathrm{L}^{-1}$ & $1.42(0.33)$ & $1.70(0.39)$ & $<0.001$ \\
\hline Triglycerides, $\mathrm{mmol} \cdot \mathrm{L}^{-1}$ & $1.40(0.81)$ & $0.99(0.48)$ & $<0.001$ \\
\hline
\end{tabular}

\section{Cardiovascular autonomic function}

Sitting

HR, bpm

$71.1(12.0) \quad 71.6(10.3) \quad 0.143$

rMSSD, ms

$21.6(13.5-30.4) \quad 24.4(16.5-35.6) \quad<0.001$

$\alpha_{1}$

$1.23(0.26)$

$1.08(0.27)$

$<0.001$

$\mathrm{BRS}, \mathrm{ms} \cdot \mathrm{mmHg}^{-1}$

$6.7(5.0-9.4)$

$6.5(4.6-8.7)$

0.054 


\section{Standing}

HR, bpm

rMSSD, ms

$\alpha_{1}$

$\mathrm{BRS}, \mathrm{ms} \cdot \mathrm{mmHg}^{-1}$
$81.6(13.3)$

$12.9(8.5-18.9)$

$1.44(0.23)$

$4.9(3.2-6.9)$
$82.8(12.2)$

0.011

$13.1(8.7-19.3) \quad 0.239$

$1.33(0.26) \quad<0.001$

$4.4(3.2-6.0) \quad 0.023$

The values are absolute or relative (\%) number of cases, means (SD) or median $\left(1^{\text {st }}-3^{\text {rd }}\right.$ quartile) and $\mathrm{p}$ value for sex-difference. $H b A l c$ glycated hemoglobin, $L D L$ low-density lipoprotein, $H D L$ high-density lipoprotein, $H R$ heart rate, $r M S S D$ root mean square of the successive differences in R-R intervals, $\alpha_{1}$ short-term fractal-like scaling exponent, $B R S$ baroreflex sensitivity 
Table 2. Cardiovascular autonomic function in the life course physical activity (PA $\left.\mathrm{P}_{\mathrm{Life}}\right)$ trajectory groups in men.

\begin{tabular}{|c|c|c|c|c|c|c|c|}
\hline \multirow[t]{3}{*}{ Men } & \multicolumn{3}{|c|}{$\mathrm{PA}_{\text {Life }}$} & \multicolumn{4}{|c|}{ Effects of PA (univariate) } \\
\hline & Active & Semi-active & Inactive & $\mathrm{p}$ Value & $\mathrm{p}$ Value & $\mathrm{p}$ Value & $\mathrm{p}$ Value \\
\hline & $\mathrm{n}=359$ & $\mathrm{n}=554$ & $\mathrm{n}=370$ & $\mathrm{PA}_{\text {Life }}$ & $\mathrm{PA}_{46 \mathrm{y}}{ }^{a}$ & $\mathrm{PA}_{31 \mathrm{y}}^{b}$ & $\mathrm{PA}_{14 \mathrm{y}}{ }^{c}$ \\
\hline \multicolumn{8}{|l|}{ Sitting } \\
\hline rMSSD, ms & $22.9(15.9-32.7)$ & $20.9(13.0-31.4)$ & $18.2(11.8-27.7)^{\dagger,+}$ & $<0.001$ & $<0.001$ & 0.024 & 0.922 \\
\hline$\alpha_{1}$ & $1.21(0.28)$ & $1.23(0.26)$ & $1.26(0.25)$ & 0.028 & $<0.001$ & 0.478 & 0.917 \\
\hline $\mathrm{BRS}, \mathrm{ms} \cdot \mathrm{mmHg}^{-1}$ & $7.5(5.4-10.0)$ & $6.9(4.8-10.1)$ & $5.9(4.8-8.5)^{\dagger}$ & $0.008^{d}$ & $<0.001^{e}$ & $0.389^{f}$ & $0.746^{g}$ \\
\hline \multicolumn{8}{|l|}{ Standing } \\
\hline rMSSD, ms & $13.6(9.4-20.1)$ & $13.2(8.6-19.5)$ & $11.5(7.8-16.9)^{\dagger, \dagger}$ & 0.002 & $<0.001$ & 0.259 & 0.651 \\
\hline$\alpha_{1}$ & $1.44(0.23)$ & $1.44(0.23)$ & $1.45(0.23)$ & 0.915 & 0.880 & 0.461 & 0.447 \\
\hline $\mathrm{BRS}, \mathrm{ms} \cdot \mathrm{mmHg}^{-1}$ & $5.5(3.7-7.3)$ & $4.8(3.0-6.8)^{*}$ & $4.3(3.0-6.1)^{*}$ & $0.011^{d}$ & $<0.001^{e}$ & $0.222^{f}$ & $0.431^{g}$ \\
\hline
\end{tabular}


Table 2. Continued.

\begin{tabular}{|c|c|c|c|c|c|c|c|}
\hline \multirow[t]{4}{*}{ Men } & \multicolumn{7}{|c|}{ Multivariate } \\
\hline & \multirow{3}{*}{$\begin{array}{c}\text { Block I } \\
\mathrm{R}^{2}\end{array}$} & \multicolumn{2}{|c|}{ Block I+PA $A_{\text {Life }}$} & \multicolumn{2}{|c|}{ Block I+II+PA $A_{\text {Life }}$} & \multicolumn{2}{|c|}{ Block I+II+PA46y } \\
\hline & & & $\mathrm{p}$ Value & & $\mathrm{p}$ Value & & $\mathrm{p}$ Value \\
\hline & & $\mathrm{R}^{2}$ & $\mathrm{PA}_{\text {Life }}$ & $\mathrm{R}^{2}$ & $\mathrm{PA}_{\text {Life }}$ & $\mathrm{R}^{2}$ & $\mathrm{PA}_{46 y}$ \\
\hline \multicolumn{8}{|l|}{ Sitting } \\
\hline $\mathrm{HR}, \mathrm{bpm}$ & 0.093 & 0.101 & $<0.001$ & 0.224 & 0.010 & 0.225 & 0.003 \\
\hline $\mathrm{rMSSD}, \mathrm{ms}$ & 0.108 & 0.110 & 0.051 & 0.185 & 0.138 & 0.192 & $<0.001$ \\
\hline$\alpha_{1}$ & 0.023 & 0.024 & 0.116 & 0.066 & 0.127 & 0.071 & 0.003 \\
\hline $\mathrm{BRS}, \mathrm{ms} \cdot \mathrm{mmHg}^{-1}$ & 0.101 & 0.103 & 0.147 & 0.219 & 0.436 & 0.222 & 0.068 \\
\hline \multicolumn{8}{|l|}{ Standing } \\
\hline $\mathrm{HR}, \mathrm{bpm}$ & 0.057 & 0.067 & $<0.001$ & 0.165 & 0.001 & 0.166 & 0.001 \\
\hline rMSSD, ms & 0.066 & 0.067 & 0.156 & 0.118 & 0.286 & 0.124 & 0.003 \\
\hline$\alpha_{1}$ & 0.001 & - & - & - & - & - & - \\
\hline $\mathrm{BRS}, \mathrm{ms} \cdot \mathrm{mmHg}^{-1}$ & 0.097 & 0.099 & 0.207 & 0.200 & 0.597 & 0.203 & 0.066 \\
\hline
\end{tabular}


The values are means (SD), median $\left(1^{\text {st }-3^{\text {rd }}}\right.$ quartile), $\mathrm{p}$ values from ANOVA or linear regression and squared $\mathrm{R}$-values from linear regression $\left(\mathrm{R}^{2}\right)$. $P A$ physical activity, $H R$ heart rate, $r M S S D$ root mean square of the successive differences in R-R intervals, $\alpha_{1}$ short-term fractal-like scaling exponent, BRS baroreflex sensitivity. ${ }^{*} \mathrm{p}<0.05$ and ${ }^{\dagger} \mathrm{p}<0.01$ compared to Active group, ${ }^{\dagger} \mathrm{p}<0.05$ compared to Semi-active group, ${ }^{a} \mathrm{n}=209 / 733 / 341$, ${ }^{b} \mathrm{n}=185 / 671 / 427,{ }^{c} \mathrm{n}=642 / 444 / 197,{ }^{d} \mathrm{n}=184 / 289 / 189,{ }^{e} \mathrm{n}=112 / 374 / 176,{ }_{\mathrm{n}}=94 / 343 / 225$, and ${ }^{g} \mathrm{n}=328 / 238 / 96$ for Active/Semi-active/Inactive PA groups. 
Table 3. Cardiovascular autonomic function in the life course physical activity (PA $\left.\mathrm{Life}_{\mathrm{f}}\right)$ trajectory groups in women.

\begin{tabular}{|c|c|c|c|c|c|c|c|}
\hline \multirow[t]{4}{*}{ Women } & \multicolumn{3}{|c|}{$\mathrm{PA}_{\text {Life }}$} & \multicolumn{4}{|c|}{ Effects of PA (univariate) } \\
\hline & \multirow[t]{2}{*}{ Active } & \multirow[t]{2}{*}{ Semi-active } & \multirow[t]{2}{*}{ Inactive } & \multirow[b]{2}{*}{$\mathrm{p}$ Value } & \multirow[b]{2}{*}{$\mathrm{p}$ Value } & \multirow[b]{2}{*}{$\mathrm{p}$ Value } & \multirow[b]{2}{*}{ p Value } \\
\hline & & & & & & & \\
\hline & $\mathrm{n}=402$ & $\mathrm{n}=908$ & $n=469$ & $\mathrm{PA}_{\text {Life }}$ & $\mathrm{PA}_{46 \mathrm{y}}{ }^{a}$ & $\mathrm{PA}_{31 \mathrm{y}}^{b}$ & $\mathrm{PA}_{14 \mathrm{y}}{ }^{c}$ \\
\hline \multicolumn{8}{|l|}{ Sitting } \\
\hline $\mathrm{HR}, \mathrm{bpm}$ & $69.6(10.5)$ & $71.7(10.0)^{\dagger}$ & $73.3(10.5)^{\dagger, \ddagger}$ & $<0.001$ & $<0.001$ & 0.012 & 0.291 \\
\hline rMSSD, ms & $28.4(19.4-40.9)$ & $24.2(16.5-35.1)^{\dagger}$ & $21.8(15.3-32.5)^{\dagger, \ddagger}$ & $<0.001$ & $<0.001$ & 0.001 & 0.426 \\
\hline$\alpha_{1}$ & $1.06(0.27)$ & $1.08(0.26)$ & $1.09(0.28)$ & 0.182 & 0.235 & 0.282 & 0.540 \\
\hline $\mathrm{BRS}, \mathrm{ms} \cdot \mathrm{mmHg}^{-1}$ & $7.3(5.0-9.4)$ & $6.3(4.4-8.5)^{*}$ & $6.4(4.6-8.3)$ & $0.032^{d}$ & $0.075^{e}$ & $0.252^{f}$ & $0.641^{g}$ \\
\hline \multicolumn{8}{|l|}{ Standing } \\
\hline $\mathrm{HR}, \mathrm{bpm}$ & $80.6(12.7)$ & $82.9(11.9)^{\dagger}$ & $84.4(12.2)^{\dagger}$ & $<0.001$ & $<0.001$ & 0.029 & 0.540 \\
\hline rMSSD, ms & $14.9(9.9-22.0)$ & $13.1(8.7-19.1)^{\dagger}$ & $11.7(7.7-17.7)^{\dagger, \dagger}$ & $<0.001$ & 0.001 & $<0.001$ & 0.311 \\
\hline$\alpha_{1}$ & $1.32(0.26)$ & $1.34(0.25)$ & $1.34(0.26)$ & 0.303 & 0.764 & 0.168 & 0.115 \\
\hline $\mathrm{BRS}, \mathrm{ms} \cdot \mathrm{mmHg}^{-1}$ & $1.59(0.54)$ & $1.44(0.50)^{\dagger}$ & $1.42(0.46)^{\dagger}$ & $0.001^{d}$ & $<0.001^{e}$ & $0.068^{f}$ & $0.422^{g}$ \\
\hline
\end{tabular}


Table 3. Continued.

\begin{tabular}{|c|c|c|c|c|c|c|c|}
\hline \multirow[t]{4}{*}{ Women } & \multicolumn{7}{|c|}{ Multivariate } \\
\hline & \multirow{3}{*}{$\begin{array}{c}\text { Block I } \\
\mathrm{R}^{2}\end{array}$} & \multicolumn{2}{|c|}{ Block I+PALife } & \multicolumn{2}{|c|}{ Block I+II+PALife } & \multicolumn{2}{|c|}{ Block I+II+PA46y } \\
\hline & & & $\mathrm{p}$ Value & & $\mathrm{p}$ Value & & $\mathrm{p}$ Value \\
\hline & & $\mathrm{R}^{2}$ & $\mathrm{PA}_{\text {Life }}$ & $\mathrm{R}^{2}$ & $\mathrm{PA}_{\text {Life }}$ & $\mathrm{R}^{2}$ & $\mathrm{PA}_{46 \mathrm{y}}$ \\
\hline \multicolumn{8}{|l|}{ Sitting } \\
\hline $\mathrm{HR}, \mathrm{bpm}$ & 0.047 & 0.057 & $<0.001$ & 0.134 & $<0.001$ & 0.135 & $<0.001$ \\
\hline $\mathrm{rMSSD}, \mathrm{ms}$ & 0.040 & 0.049 & $<0.001$ & 0.095 & $<0.001$ & 0.090 & 0.032 \\
\hline$\alpha_{1}$ & 0.018 & - & - & - & - & - & - \\
\hline $\mathrm{BRS}, \mathrm{ms} \cdot \mathrm{mmHg}^{-1}$ & 0.047 & 0.050 & 0.140 & 0.142 & 0.257 & 0.142 & 0.359 \\
\hline \multicolumn{8}{|l|}{ Standing } \\
\hline $\mathrm{HR}, \mathrm{bpm}$ & 0.024 & 0.032 & $<0.001$ & 0.105 & 0.001 & 0.107 & $<0.001$ \\
\hline rMSSD, ms & 0.022 & 0.033 & $<0.001$ & 0.074 & $<0.001$ & 0.068 & 0.028 \\
\hline$\alpha_{1}$ & 0.006 & - & - & - & - & - & - \\
\hline $\mathrm{BRS}, \mathrm{ms} \cdot \mathrm{mmHg}^{-1}$ & 0.082 & 0.088 & 0.016 & 0.188 & 0.037 & 0.187 & 0.068 \\
\hline
\end{tabular}


The values are means(SD), median $\left(1^{\text {st }}-3^{\text {rd }}\right.$ quartile), p-values from ANOVA or linear regression and squared $\mathrm{R}$-values from linear regression $\left(\mathrm{R}^{2}\right)$. $P A$ physical activity, $H R$ heart rate, $r M S S D$ root mean square of the successive differences in R-R intervals, $\alpha_{1}$ short-term fractal-like scaling exponent, BRS baroreflex sensitivity. ${ }^{*} \mathrm{p}<0.05$ and ${ }^{\dagger} \mathrm{p}<0.01$ compared to Active group, ${ }^{\ddagger} \mathrm{p}<0.05$ and ${ }^{\S} \mathrm{p}<0.01$ compared to Semi-active group, ${ }^{a} \mathrm{n}=302 / 1077 / 400,{ }^{b} \mathrm{n}=204 / 1032 / 543,{ }^{c} \mathrm{n}=496 / 766 / 517,{ }^{d} \mathrm{n}=191 / 407 / 209,{ }^{e} \mathrm{n}=147 / 489 / 171,{ }_{\mathrm{n}}=98 / 477 / 232,{ }^{g} \mathrm{n}=233 / 357 / 217$ for Active/Semiactive/Inactive PA groups. 
Figure 1.
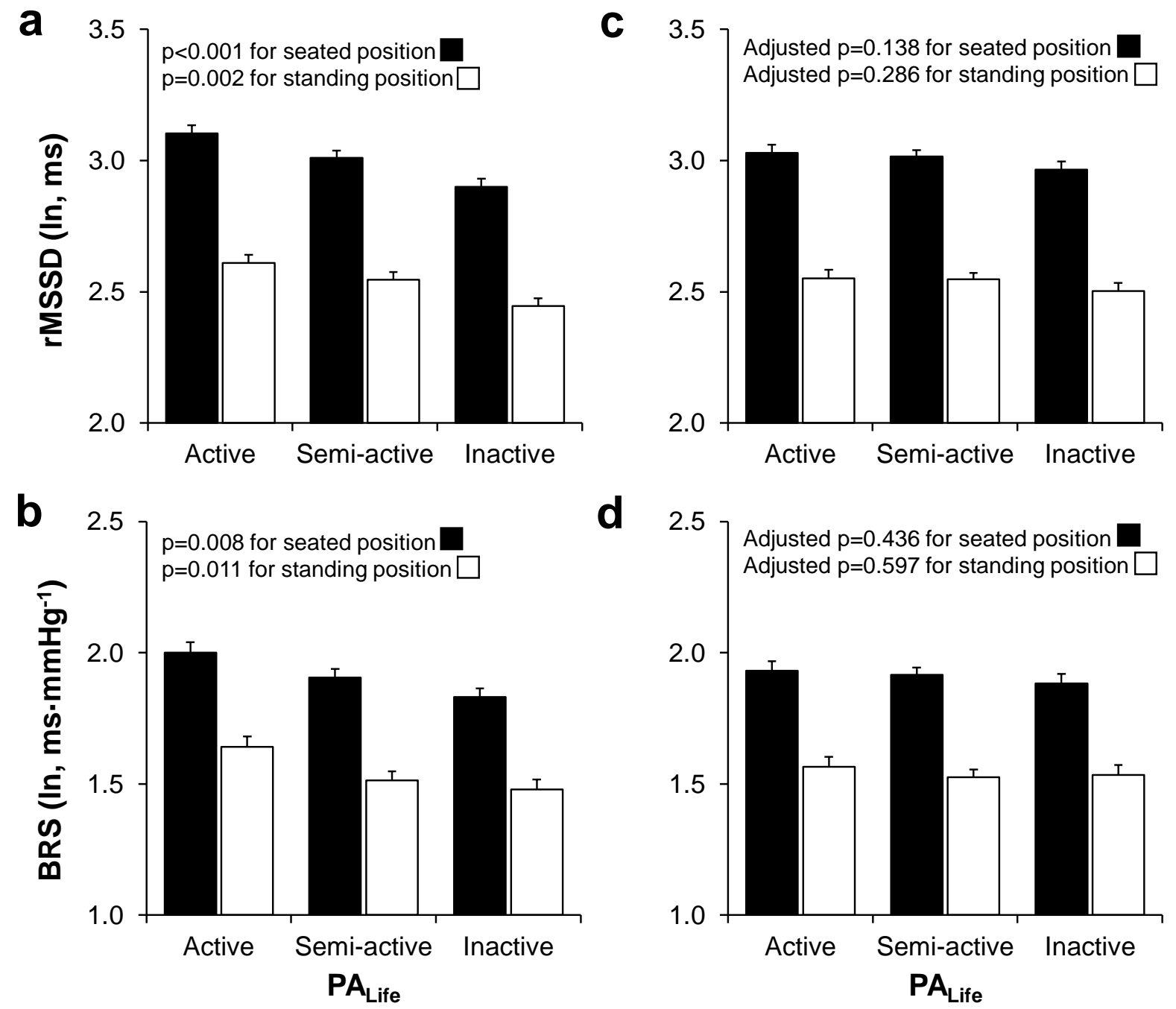
Figure 2.
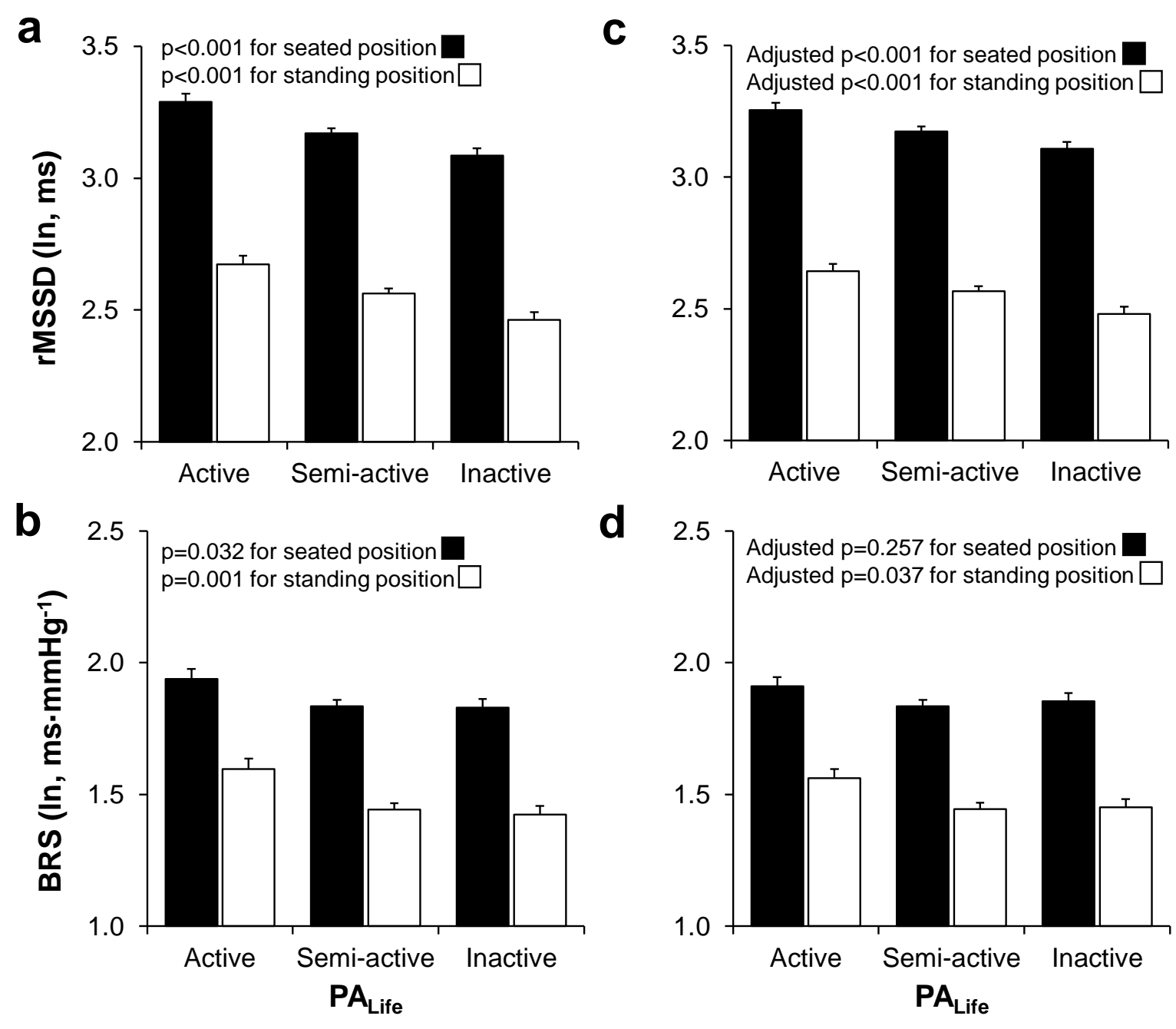
Supplementary table 1. Age-specific frequency of brisk physical activity (PA) and its changes according to the lifelong physical activity (PA $\mathrm{Aife}_{\mathrm{f}}$.

\begin{tabular}{|c|c|c|c|c|c|c|}
\hline \multirow[b]{2}{*}{ PAlife } & \multicolumn{3}{|c|}{ Men } & \multicolumn{3}{|c|}{ Women } \\
\hline & Active & Semi-active & Inactive & Active & Semi-active & Inactive \\
\hline & $n=359$ & $\mathrm{n}=554$ & $n=370$ & $n=402$ & $\mathrm{n}=908$ & $n=469$ \\
\hline $\mathbf{P A}_{14 \mathbf{y}}{ }^{*}$ 3-7 times per week & $287(80)$ & $235(42)$ & $120(32)$ & $221(55)$ & $189(21)$ & $18(5)$ \\
\hline Twice per week & $50(14)$ & $248(45)$ & $146(40)$ & $128(32)$ & $462(51)$ & $176(38)$ \\
\hline$<1$ per week & $22(6)$ & $71(13)$ & $104(28)$ & $53(13)$ & $257(28)$ & $207(44)$ \\
\hline PA31y ${ }^{*}$ 4-7 times per week & $162(45)$ & $3(0.5)$ & $20(5)$ & $175(44)$ & $2(0.2)$ & $27(6)$ \\
\hline 1-3 times per week & $196(55)$ & $423(76)$ & $52(14)$ & $222(55)$ & $720(79)$ & $90(19)$ \\
\hline$<1$ per week & $1(0.3)$ & $128(23)$ & $298(81)$ & $5(1)$ & $186(21)$ & $352(75)$ \\
\hline $\mathbf{P A}_{46 \mathbf{y}}{ }^{*}$ 4-7 times per week & $153(43)$ & $36(7)$ & $20(5)$ & $219(55)$ & $51(6)$ & $32(7)$ \\
\hline 1-3 times per week & $204(57)$ & $417(75)$ & $112(30)$ & $181(45)$ & 745 (82) & $151(32)$ \\
\hline$<1$ per week & $2(0.6)$ & $101(18)$ & $238(64)$ & $2(0.5)$ & $112(12)$ & $286(61)$ \\
\hline PAChange14-31y ${ }^{*}$ & $57(16)$ & $56(10)$ & $32(9)$ & $112(28)$ & $208(23)$ & $64(14)$ \\
\hline No change & $128(36)$ & $221(40)$ & $102(28)$ & $149(37)$ & $431(48)$ & $192(41)$ \\
\hline Decrease & $174(49)$ & $277(50)$ & $236(64)$ & $141(35)$ & $269(30)$ & $213(45)$ \\
\hline
\end{tabular}




$\begin{array}{rrrrrrr}\text { PA }_{\text {Change31-46y }}{ }^{*} & \text { Increase } & 88(25) & 135(24) & 130(35) & 144(36) & 202(22) \\ \text { No change } & 174(49) & 333(60) & 170(46) & 158(39) & 612(67) & 179(38) \\ \text { Decrease } & 97(27) & 86(16) & 70(19) & 100(25) & 94(10)\end{array}$

The values are absolute or relative (\%) number of cases. ${ }^{*} \mathrm{p}<0.001$ for the main effect by Chi-square. 
Supplementary table 2. The most significant determinants of cardiovascular autonomic function in the multivariate linear regression in men.

First, the significant independent factors in Block I were established (stepwise, forward). Thereafter, the significant factors in Block II (stepwise, forward) were added to the initially established model (Block I).

\begin{tabular}{|c|c|c|c|c|c|c|c|c|c|c|c|c|}
\hline \multirow[t]{3}{*}{ Men } & \multicolumn{4}{|c|}{$\overline{\mathrm{HR}}$} & \multicolumn{4}{|c|}{ rMSSD, ln } & \multicolumn{4}{|c|}{ BRS, ln } \\
\hline & \multicolumn{2}{|c|}{ Sitting } & \multicolumn{2}{|c|}{ Standing } & \multicolumn{2}{|c|}{ Sitting } & \multicolumn{2}{|c|}{ Standing } & \multicolumn{2}{|c|}{ Sitting } & \multicolumn{2}{|c|}{ Standing } \\
\hline & $\beta$ & $\mathrm{p}$ Value & $\beta$ & $\mathrm{p}$ Value & $\beta$ & $\mathrm{p}$ Value & $\beta$ & $\mathrm{p}$ Value & $\beta$ & $\mathrm{p}$ Value & $\beta$ & $\mathrm{p}$ Value \\
\hline \multicolumn{13}{|l|}{ Block I } \\
\hline $\mathrm{PA}_{\text {Life }}$ & -1.09 & 0.008 & -1.59 & 0.001 & 0.03 & 0.141 & - & - & - & - & - & - \\
\hline Smoking status & 3.95 & $<0.001$ & 3.87 & $<0.001$ & -0.24 & $<0.001$ & -0.30 & $<0.001$ & -0.18 & 0.001 & -0.19 & 0.001 \\
\hline Alcohol consumption & 1.90 & 0.089 & 2.95 & 0.022 & -0.12 & 0.044 & - & - & - & - & - & - \\
\hline Sufficiency of sleep & 1.60 & 0.015 & 1.51 & 0.046 & -0.09 & 0.015 & -0.10 & 0.007 & - & - & - & - \\
\hline Body mass index & -0.13 & 0.757 & -0.41 & 0.302 & -0.06 & 0.001 & -0.04 & 0.038 & -0.03 & 0.316 & -0.03 & 0.267 \\
\hline \multicolumn{13}{|l|}{ Block II } \\
\hline Systolic blood pressure & -3.10 & $<0.001$ & -4.03 & $<0.001$ & 0.09 & 0.001 & 0.12 & $<0.001$ & - & - & 0.09 & 0.006 \\
\hline Diastolic blood pressure & 5.81 & $<0.001$ & 6.40 & $<0.001$ & -0.22 & $<0.001$ & -0.22 & $<0.001$ & -0.17 & $<0.001$ & -0.24 & $<0.001$ \\
\hline Waist-hip-ratio & 0.90 & 0.028 & - & 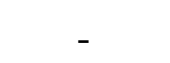 & - & - & - & - & -0.06 & 0.012 & -0.06 & 0.036 \\
\hline
\end{tabular}




\begin{tabular}{|c|c|c|c|c|c|c|c|c|c|c|c|c|}
\hline Plasma glucose & 1.23 & $<0.001$ & 1.27 & 0.001 & -0.04 & 0.010 & & - & -0.06 & 0.004 & -0.05 & 0.013 \\
\hline Total cholesterol & - & - & - & - & -0.04 & 0.031 & -0.06 & 0.001 & - & - & -0.04 & 0.039 \\
\hline Triglycerides & 1.11 & 0.001 & 1.15 & 0.002 & -0.05 & 0.003 & - & - & - & - & - & - \\
\hline
\end{tabular}

The values are unstandardized coefficients $\beta$ (per class or $1 \mathrm{SD}$ ) and their significances (p value). HR heart rate, $r M S S D$ root mean square of the successive differences in R-R intervals, $B R S$ baroreflex sensitivity, $P A$ physical activity. 
Supplementary table 3. The most significant determinants of cardiovascular autonomic function in the multivariate linear regression in women.

First, the significant independent factors in Block I were established (stepwise, forward). Thereafter, the significant factors in Block II (stepwise, forward) were added to the initially established model (Block I).

\begin{tabular}{|c|c|c|c|c|c|c|c|c|c|c|c|c|}
\hline \multirow[t]{3}{*}{ Women } & \multicolumn{4}{|c|}{ HR } & \multicolumn{4}{|c|}{ rMSSD, ln } & \multicolumn{4}{|c|}{ BRS, ln } \\
\hline & \multicolumn{2}{|c|}{ Sitting } & \multicolumn{2}{|c|}{ Standing } & \multicolumn{2}{|c|}{ Sitting } & \multicolumn{2}{|c|}{ Standing } & \multicolumn{2}{|c|}{ Sitting } & \multicolumn{2}{|c|}{ Standing } \\
\hline & $\beta$ & $\mathrm{p}$ Value & $\beta$ & $\mathrm{p}$ Value & $\beta$ & $\mathrm{p}$ Value & $\beta$ & $\mathrm{p}$ Value & $\beta$ & $\mathrm{p}$ Value & $\beta$ & $\mathrm{p}$ Value \\
\hline \multicolumn{13}{|l|}{ Block I } \\
\hline $\mathrm{PA}_{\text {Life }}$ & -1.20 & $<0.001$ & -1.19 & 0.003 & 0.07 & $<0.001$ & 0.08 & $<0.001$ & - & - & 0.05 & 0.041 \\
\hline Alcohol consumption & 1.85 & 0.037 & 2.24 & 0.036 & -0.04 & 0.433 & - & - & - & - & -0.07 & 0.266 \\
\hline Body mass index & 0.56 & 0.031 & 0.19 & 0.553 & -0.05 & 0.002 & -0.04 & 0.013 & -0.03 & 0.11 & -0.06 & 0.001 \\
\hline \multicolumn{13}{|l|}{ Block II } \\
\hline Systolic blood pressure & -2.91 & $<0.001$ & -4.64 & $<0.001$ & 0.10 & $<0.001$ & 0.13 & $<0.001$ & - & - & 0.07 & 0.013 \\
\hline Diastolic blood pressure & 4.61 & $<0.001$ & 5.74 & $<0.001$ & -0.19 & $<0.001$ & -0.17 & $<0.001$ & -0.14 & $<0.001$ & -0.21 & $<0.001$ \\
\hline Waist-hip-ratio & - & - & - & - & - & - & 0.03 & 0.032 & - & - & - & - \\
\hline Plasma glucose & - & - & - & - & - & - & - & - & -0.04 & 0.013 & -0.04 & 0.01 \\
\hline Total cholesterol & - & - & - & - & - & - & - & - & - & - & -0.05 & 0.001 \\
\hline
\end{tabular}


HDL cholesterol

Triglycerides

$-\quad-$

1.33
$<0.001$

$-0.15$

$\begin{array}{ccccc}- & -0.03 & 0.048 & -0.03 & 0.032 \\ <0.001 & -0.06 & <0.001 & -0.09 & <0.001\end{array}$

The values are unstandardized coefficients $\beta$ (per class or 1 SD) and their significances (p value). HR heart rate, $r M S S D$ root mean square of the successive differences in R-R intervals, $B R S$ baroreflex sensitivity, $P A$ physical activity, $H D L$ high-density lipoprotein. 